\title{
Corrigendum
}

\section{Obésité et vieillissement en santé : bien-être social, fonctionnel et mental chez les personnes âgées au Canada}

Ce corrigendum vise à corriger un certain nombre d'erreurs ou d'imprécisions, ayant paru aux pages 491 à 497 de l'article suivant :

Rao DP, Patel P, Roberts KC, Thompson W. Obésité et vieillissement en santé : bien-être social, fonctionnel et mental chez les personnes âgées au Canada. Promotion de la santé et prévention des maladies chroniques au Canada. 2018;38(12):491-499. doi: 10.24095 /hpcdp.38.12.01f.

\section{1. p. 491 (Résumé, Résultats)}

\section{Avant la correction}

Bien que le bonheur et la satisfaction à l'égard de la vie ne soient pas associés à l'obésité, les femmes âgées souffrant d'obésité ont déclaré avoir une perception défavorable de leur état de santé.

\section{Après la correction}

Bien que le bonheur et la satisfaction à l'égard de la vie ne soient pas associés à l'obésité, les femmes âgées souffrant d'obésité ont déclaré, de façon plus prononcée que les hommes, avoir une perception défavorable de leur état de santé.

\section{2. p. 493 (Méthodologie, Variables, Santé mentale et bien-être)}

\section{Avant la correction}

Nous avons déterminé l'état de santé mentale en fonction de l'autodéclaration d'un trouble de l'humeur ou d'un trouble d'anxiété diagnostiqué par un médecin. Plusieurs mesures ont été utilisées pour évaluer le bien-être mental. Nous avons défini le bonheur comme le sentiment d'être heureux trois jours ou plus par semaine, par opposition à moins de trois jours. Bien que cette mesure ne remplace pas une évaluation de la santé mentale en bonne et due forme, son utilité a été confirmée pour la mesure de l'état de santé mentale général ${ }^{28}$. Le bonheur autodéclaré est associé à une réduction de la mortalité, et peut être médié par l'activité physique et la comorbidité chez les personnes âgées ${ }^{29}$. La satisfaction à l'égard de la vie a été évaluée à partir des réponses indiquant une certaine satisfaction à l'égard de la vie par opposition aux réponses neutres ou traduisant une insatisfaction. Ces variables (autoévaluation de la santé mentale et autoévaluation du vieillissement en santé) ont été codées sous forme binaire (état " passable » et " mauvais » par opposition à " bon ", " très bon » et « excellent»).

\section{Après la correction}

Nous avons déterminé l'état de santé mentale en fonction de l'autodéclaration d'un trouble de l'humeur ou d'un trouble d'anxiété diagnostiqué par un médecin. Plusieurs mesures ont été utilisées pour évaluer le bien-être mental. Nous avons défini le bonheur comme le sentiment d'être heureux trois jours ou plus par semaine, par opposition à moins de trois jours. Le bonheur autodéclaré est associé à une réduction de la mortalité, et peut être médié par l'activité physique et la comorbidité chez les personnes âgées ${ }^{29}$. La satisfaction à l'égard de la vie a été évaluée à partir des réponses indiquant une certaine satisfaction à l'égard de la vie par opposition aux réponses neutres ou traduisant une insatisfaction. On a posé aux participants les deux questions suivantes afin d'évaluer la santé mentale et le vieillissement en santé, respectivement : "En général, diriez-vous que votre santé mentale est excellente, très bonne, bonne, passable ou mauvaise? » et « Pour ce qui est de votre propre vieillissement en santé, diriez-vous qu'il est excellent, très bon, bon, passable ou mauvais? » Bien que l'autoévaluation de la santé mentale ne remplace pas une évaluation de la santé mentale en bonne et due forme, son utilité a été confirmée pour la mesure de l'état de santé mentale général ${ }^{28}$. Ces variables (autoévaluation de la santé mentale et autoévaluation du vieillissement en santé) ont été codées sous forme binaire (état " passable » et " mauvais » par opposition à " bon », " très bon » et " excellent »). 


\section{3. p. 493 (Résultats, $2^{e}$ paragraphe)}

\section{Avant la correction}

La proportion de participants ayant déclaré consommer 4 boissons alcoolisées ou plus par semaine variait de façon significative entre les sexes et elle diminuait significativement en fonction de l'âge. La fréquence de l'obésité était significativement plus élevée chez les hommes et diminuait avec l'âge jusqu'au groupe d'âge des 75 à 85 ans. Les femmes de 75 à 85 ans étaient plus nombreuses que les hommes à souffrir d'obésité, en dépit du fait qu'elles présentaient une diminution de l'obésité avec l'âge. Enfin, les femmes étaient significativement plus nombreuses que les hommes à présenter une multimorbidité entre 55 et 64 ans, différence qui n’a pas été observée dans le groupe d'âge des 75 à 85 ans (tableau 1).

\section{Après la correction}

La proportion de participants ayant déclaré consommer 4 boissons alcoolisées ou plus par semaine variait de façon significative entre les sexes et entre les différents groupes d’âge. En ce qui a trait à l'obésité, des différences statistiquement significatives ont été observées entre les différents groupes d'âge, chez les hommes comme chez les femmes. La fréquence de l'obésité était significativement plus élevée chez les hommes que chez les femmes chez les 55 à 64 ans, alors que, chez les 75 à 85 ans, la prévalence de l’obésité était plus élevée chez les femmes que chez les hommes, mais ce dernier résultat n'était pas statistiquement significatif. Enfin, les femmes étaient significativement plus nombreuses que les hommes à présenter une multimorbidité entre 55 et 64 ans, différence qui n'a pas été observée dans le groupe d'âge des 75 à 85 ans (tableau 1).

\section{4. p. 493 à 495 (Résultats, $3^{e}$ paragraphe)}

\section{Avant la correction}

La réduction du fonctionnement physique s'est révélée fortement associée à l'obésité tant chez les hommes que chez les femmes, avec des différences significatives entre les sexes uniquement chez les 65 à 74 ans. La force de cette association entre la réduction du fonctionnement physique et l'obésité augmentait avec l'âge chez les deux sexes. Nous avons de la même manière constaté une association significative entre les limitations dans la vie quotidienne et l'obésité chez les deux sexes, la force de cette association augmentant avec l'âge. La différence entre les sexes était significative dans tous les groupes d’âge, les femmes souffrant d'obésité ayant déclaré plus de limitations que les hommes souffrant d'obésité.

\section{Après la correction}

La réduction du fonctionnement physique s'est révélée fortement associée à l'obésité tant chez les hommes que chez les femmes, avec des différences significatives entre les sexes uniquement chez les 65 à 74 ans. Nous avons de la même manière constaté une association significative entre les limitations dans la vie quotidienne et l'obésité chez les deux sexes. La différence entre les sexes était significative dans tous les groupes d’âge, les femmes souffrant d'obésité ayant déclaré plus de limitations que les hommes souffrant d'obésité.

\section{5. p. 495 (Analyse, $1^{\text {er }}$ paragraphe)}

\section{Avant la correction}

Cependant, alors que certaines études laissent penser qu'une situation financière précaire est associée à la maladie ${ }^{32}$, nous avons observé que les participants de l’ELCV bénéficiaient d'une situation financière autoévaluée solide.

\section{Après la correction}

Nous avons observé, parmi les participants de l'ELCV de tous les groupes d'âge, qu'une proportion plus faible de femmes que d'hommes avait un revenu personnel égal ou supérieur à 50000 \$ ou résidait dans son propre logement. Or une situation financière précaire a été associée à la maladie ${ }^{32}$. Nous avons également observé une prévalence plus élevée de multimorbidité chez les femmes que chez les hommes, et ce, dans tous les groupes d’âge, sauf le groupe le plus âgé.

\section{6. p. 497 (Analyse, $4^{e}$ paragraphe)}

\section{Avant la correction}

La perception défavorable qu'ont les femmes âgées de leur santé mentale est notable, même si elle s'améliore avec l'âge.

\section{Après la correction}

La perception défavorable qu'ont les femmes âgées souffrant d’obésité de leur santé mentale est notable. 